\title{
The Effect of External Gaseous Environments on the Photoluminescence Intensity of Quantum-Dimensional Composite System
}

\author{
V. V. Danilov, ${ }^{1,2}$ A. I. Khrebtov, ${ }^{3}$ I. V. Shtrom, ${ }^{3,4,5}$ G. E. Cirlin, ${ }^{3,4,5,6}$ and Yu B. Samsonenko ${ }^{3,5}$ \\ ${ }^{1}$ Vavilov State Optical Institute, Saint Petersburg 199034, Russia \\ ${ }^{2}$ Saint Petersburg State Transport University, Saint Petersburg 191031, Russia \\ ${ }^{3}$ Saint Petersburg Academic University-Nanotechnology Research and Education Center, Russian Academy of Sciences, \\ Saint Petersburg 194021, Russia \\ ${ }^{4}$ Fock Institute of Physics, Saint Petersburg State University, Saint Petersburg 198504, Russia \\ ${ }^{5}$ Institute for Analytical Instrumentation, Russian Academy of Sciences, Saint Petersburg 198095, Russia \\ ${ }^{6}$ Saint Petersburg State Polytechnic University, Saint Petersburg 195251, Russia
}

Correspondence should be addressed to V. V. Danilov; vdanilov039@gmail.com

Received 27 August 2015; Revised 5 December 2015; Accepted 10 December 2015

Academic Editor: Paulo Cesar Morais

Copyright (C) 2015 V. V. Danilov et al. This is an open access article distributed under the Creative Commons Attribution License, which permits unrestricted use, distribution, and reproduction in any medium, provided the original work is properly cited.

\begin{abstract}
The luminescence properties of the composite system based on colloidal CdSe/ZnS core shell quantum dots and GaAs nanowires under continuous laser irradiation are investigated. The phenomenon of photoinduced luminescence enhancement under various gaseous environments is shown. In addition to the previously reported mechanisms of the photoluminescence enhancement, a new one which is connected to the quantum dots losses of vibrational energy during the collisions with molecules of external gases is proposed.
\end{abstract}

\section{Introduction}

Inorganic hybrid nanostructures containing two or more nanocomponents have attracted significant attention in recent years due to their great potential for functional nanoscale electronic and optical devices [1]. For example, structures that combine the nanowires (NWs) and colloidal quantum dots (QDs) are expected to have more functionalities and superior properties providing applications in many practical devices, such as photodetectors and solar cells [2-4]. Recently, in [5] we have demonstrated the possibility of fabricating a composite system based on colloidal CdSe/ZnS core shell QDs deposited onto an array of GaAs NWs. Compared with planar substrates the developed surface of NWs array is provided with the retention of a large number of QDs and as a result with an increase of the photoluminescence (PL) efficiency of QDs per unit area as well as a temperature stability of PL. At the same time, the luminescence quantum yield of
QDs depends on many factors (including technological) that may lead to decrease of the quantum yield. In our case, the emissivity of the investigated composite structure significantly depends on external factors, as evidenced by our discovery of the effects of PL enhancement at the ambient conditions and PL quenching under vacuum. A significant number of studies have been reported where the photoinduced fluorescence enhancement behavior of CdSe/ZnS QDs under gaseous environments was investigated. However, a consensus on the PL enhancement mechanism has not been reached since different conditions have been used in these studies (e.g., gaseous environments, ligands, and various substrates). Previously proposed mechanisms include passivation of surface states by photoabsorbed molecules [6,7], photoneutralization of local charged centers [8], photoionization of QDs [9], and photoinduced structure transformation of QD surface [10]. To understand the origin of the effects observed and fabricate more effective emitters based on previously 
proposed hybrid system it is necessary to investigate PL kinetics peculiarities at different gaseous environment in a view of surface photochemistry and optical properties of this composite material. In this paper, we carried out the detailed study of the PL kinetics of the composite structure in a vacuum and at the presence of various gaseous environments (air, nitrogen, and xenon) under laser excitation.

\section{Materials and Methods}

The composite structures were fabricated following procedure described in [5]. Briefly, at the first stage, GaAs NWs were grown on GaAs (111)B substrate in an EP-1203 molecular-beam epitaxy system [11]. The average height and diameter of the NWs are $250 \mathrm{~nm}$ and $20 \mathrm{~nm}$, respectively, and the array density is $10^{9} \mathrm{~cm}^{-2}$. Then, a solution of QDs in toluene with a concentration of about $10^{-6} \mathrm{M}$ was deposited onto the substrate with grown NWs using micropipette. From the HRTEM analysis, as was shown in [5], entire surface of the NW is covered by a uniformly thick ( $15 \mathrm{~nm})$ amorphous layer consisting of an array of QDs attached to the NW surface via trioctylphosphine oxide (TOPO) molecules. The average spacing between the QD centers is $6 \mathrm{~nm}$. The optical properties of the NW/QD hybrid system were studied using a PL setup based on a monochromator with a focal length of $0.5 \mathrm{~m}$ and an aperture ratio of $1 / 6.5$ equipped with a diffraction grating with 600 grooves $/ \mathrm{mm}$. The PL was excited by continuous radiation from an argon laser $(488 \mathrm{~nm})$ or a helium-cadmium laser $(325 \mathrm{~nm})$ focused onto the sample surface in spot $1 \mathrm{~mm}$ diameter. The density of laser power was $0.1-1 \mathrm{~W} / \mathrm{cm}^{2}$. To study the PL kinetics upon gaseous environment the sample was placed in a glass bulb which was pumped down to $10^{-4}$ Torr and then filled with the gas.

\section{Results and Discussion}

Figure 1 demonstrates a plot of luminescence intensity in time for the QDs solution in toluene showing the absence of significant changes during the whole time of registration. The inset presents the luminescence spectra of the composite structure coincident with the spectrum of QDs in solution and almost independent of environmental conditions (ambient or vacuum). An important feature of the structure of the investigated composite system is the uniform distribution of QDs on the surface of the NWs and the absence of QDs agglomerates that could result in a change of the fluorescence spectrum. As mentioned above, in our case the evolution of PL intensity at ambient and in vacuum conditions has opposite behavior: enhancement or quenching, correspondingly (Figure 2). The effect is completely reversible which is a significant argument in favor of its nonchemical nature.

The time dependence of PL increase can be described by biexponential function $y=y_{0}+A_{1} e^{-\left(t-t_{0}\right) / \tau_{1}}+A_{2} e^{-\left(t-t_{0}\right) / \tau_{2}}$ with $\tau_{1} \sim 10 \mathrm{sec}$ and $\tau_{2} \sim 90 \mathrm{sec}$. The PL decay curve in a vacuum condition has biexponential behavior, also with decay times of $\tau_{1} \sim 5 \mathrm{sec}$ and $\tau_{2} \sim 35 \mathrm{sec}$. The behavior of PL increasing in the presence of gases with different chemical and kinetic characteristics (xenon, dry nitrogen, and air) has

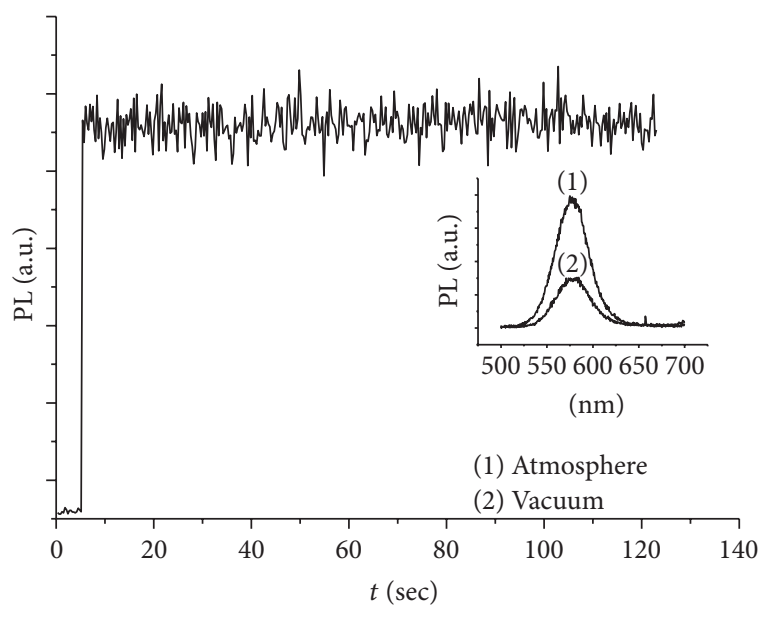

FIgURE 1: The time dependence of luminescence intensity of QDs solution in toluene. The inset presents the luminescence spectra of the composite structure.

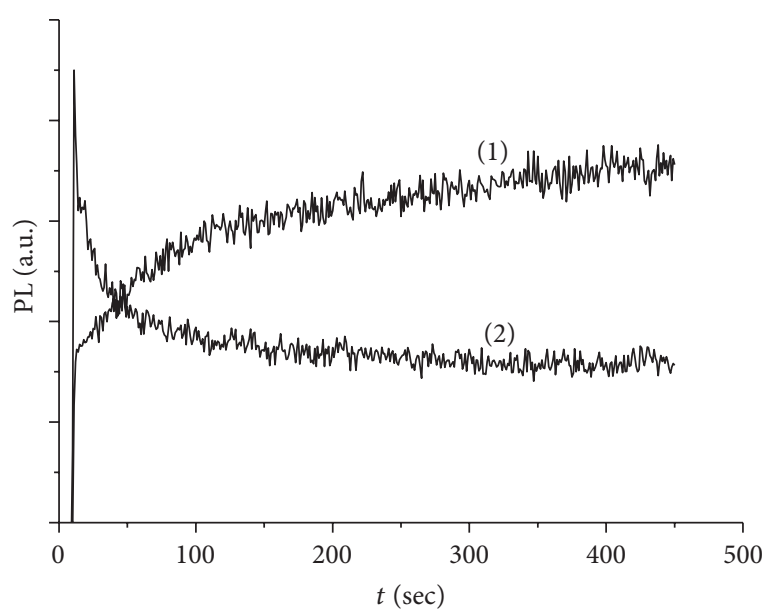

(1) Atmosphere

(2) Vacuum

FIgURE 2: PL intensity as a function of laser illumination time at the ambient conditions (1) and in a vacuum (2).

a similar trend (Figure 3). Therefore, the effect observed cannot be easily explained by the passivation of the QD trap states by photoadsorbed molecules but can also be attributed to the Arrhenius (thermal) stimulation of the exciton recombination in QDs.

To interpret our dependencies, we used the results of the study of the effect of foreign gases on the fluorescence of complex molecules [12-15]. In [12] it was shown that addition of the foreign gases to the complex molecules in vapour under light excitation around the Stokes lines range results in the fluorescence enhancement. The effect consists in the fluorescence increase of a complex molecule as a result of its vibrational deactivation by inelastic collisions with molecules of foreign gases following light absorption. Energy transfer process is characterised by the values of the amount of vibrational energy of the excited molecules lost in collisions, as well as 
TABLE 1: Van-der-Waals constants $k$, nonradiative recombination energies $\varphi$, accommodation coefficients $\alpha$, and times of increasing and decreasing of luminescence for various gases.

\begin{tabular}{|c|c|c|c|c|c|}
\hline \multirow[t]{2}{*}{ External gases } & \multirow{2}{*}{$\begin{array}{l}\text { Van-der-Waals constant } k \text {, } \\
\qquad\left(\mathrm{kg} \cdot \mathrm{M}^{4}\right) /\left(\mathrm{Mol} \cdot \mathrm{sec}^{2}\right)\end{array}$} & \multirow[t]{2}{*}{$\varphi$} & \multirow[t]{2}{*}{ Accommodation coefficient, $\alpha$} & \multicolumn{2}{|c|}{$\begin{array}{l}\text { Times of increasing and decreasing (for vacuum) } \\
\text { of luminescence }\end{array}$} \\
\hline & & & & $\tau_{1}, \sec$ & $\tau_{2}, \mathrm{sec}$ \\
\hline $\mathrm{Xe}$ & 0.419 & $55.2 / 41$ & 0.38 & $13.8 \pm 2.6$ & $108 \pm 16$ \\
\hline $\mathrm{N}_{2}$ (dry) & 0.139 & $55.1 / 37$ & 0.29 & $17.6 \pm 3.3$ & $133 \pm 20$ \\
\hline \multicolumn{6}{|l|}{ Air } \\
\hline $\mathrm{H}_{2} \mathrm{O}$ & 0.546 & & & & \\
\hline $\mathrm{O}_{2}$ & 0.136 & $55.5 / 49$ & 0.59 & $9.4 \pm 1.8$ & $83 \pm 11$ \\
\hline $\mathrm{CO}_{2}$ & 0.361 & & & & \\
\hline Vacuum & & & & $5 \pm 1$ & $35 \pm 5$ \\
\hline
\end{tabular}

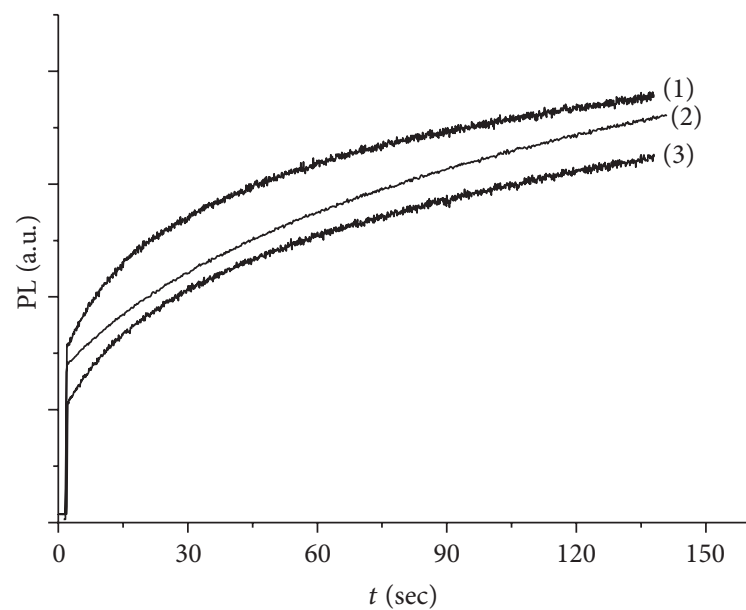

(1) Atmosphere

(2) $\mathrm{Xe}$

FIGURE 3: PL intensity as a function of laser irradiation time under various gaseous environments ((1) atmosphere, (2) Xe, and (3) $\mathrm{N}_{2}$ ).

by the value of the dimensionless accommodation coefficient $\alpha$ entered by Neporent and Mirumyants as a measure of the efficiency of energy transfer [12].

The values of accommodation coefficients $\alpha$, times of increasing and decreasing fluorescence $\tau_{1}$ and $\tau_{2}$, nonradiative recombination energies $\varphi$, and Van-der-Waals constants of different gases for our case are listed in Table 1. The increasing luminescence times given in Table 1 for dry nitrogen and air containing water vapour differ significantly. At the same time there is an obvious correlation between the times of increasing luminescence, Van-der-Waals constants, and accommodation coefficients for $\mathrm{Xe}, \mathrm{N}_{2}$, and $\mathrm{H}_{2} \mathrm{O}$. As follows from our experimental data (Figure 3 ) the value of additionally illuminated energy of luminescence (before reaching saturation) is approximately equal for all cases of external gases. At the same time it is proportional to amount of radiationless deactivated energy during collisions energy $\varphi$. The latter is in turn proportional to the product $\varphi \sim \alpha \tau$. The observed coincidence of the values of $\varphi$ for all considered cases speaks in favor of our interpretation allowing for significant influence

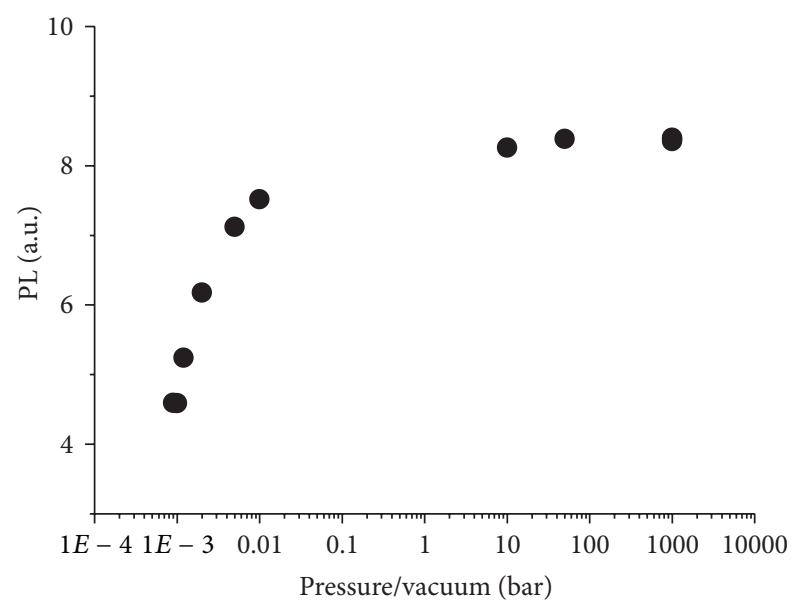

FIGURE 4: PL intensity as a function of air pressure.

of nonphotochemical factors on the photoluminescence kinetics of our hybrid system. The dependence of luminescence intensity of environment pressure ranging from $10^{-4}$ Torr to $1 \mathrm{~atm}$ obtained in our experiments (Figure 4) is in agreement with previous considerations.

The PL decay under vacuum conditions can be explained by the fact that in the absence of external gases the continuous laser radiation leads to the increase of the excess of vibrational energy in QDs. As shown in [12] this growth has a significant impact on the reduction of the quantum yield and kinetic characteristics of luminescence.

In conclusion, the authors thank V. Talalaev for photoluminescence measurements.

\section{Conclusion}

In summary, we study the effect of the enhancement of luminescence of the composite system based on colloidal $\mathrm{CdSe} / \mathrm{ZnS}$ core shell quantum dots and GaAs nanowires at the presence of external gases under continuous light illumination. A model based on the losing of vibrational energy from quantum dots in collisions with external gases molecules is proposed to describe the evolution process of enhancement of the luminescence. We also note that 
the results demonstrated in this work do not contradict the different mechanisms proposed previously but show a possibility of another parallel mechanism of the luminescence increase of the quantum dots.

\section{Conflict of Interests}

The authors declare no conflict of interests regarding the publication of this paper.

\section{Acknowledgment}

The experimental samples examined in this paper were prepared under the support of Russian Science Foundation, Grant 14-12-00393.

\section{References}

[1] T.-D. Nguyen, "Portraits of colloidal hybrid nanostructures: controlled synthesis and potential applications," Colloids and Surfaces B: Biointerfaces, vol. 103, pp. 326-344, 2013.

[2] M. L. Lu, C. H. Lin, and Y. F. Chen, "Enhanced photocurrent gain and spectrum range based on the composite consisting of $\mathrm{SnO}_{2}$ nanowires and CdSe quantum dots," Applied Physics Letters, vol. 99, no. 8, Article ID 081109, 2011.

[3] M.-L. Lu, C.-W. Lai, H.-J. Pan, C.-T. Chen, P.-T. Chou, and Y.F. Chen, "A facile integration of zero (I-III-VI quantum dots) and one (single $\mathrm{SnO}_{2}$ nanowire) dimensional nanomaterials: fabrication of a nanocomposite photodetector with ultrahigh gain and wide spectral response," Nano Letters, vol. 13, no. 5, pp. 1920-1927, 2013.

[4] J. Briscoe, D. E. Gallardo, S. Hatch, V. Lesnyak, N. Gaponik, and S. Dunn, "Enhanced quantum dot deposition on $\mathrm{ZnO}$ nanorods for photovoltaics through layer-by-layer processing," Journal of Materials Chemistry, vol. 21, no. 8, pp. 2517-2523, 2011.

[5] A. I. Khrebtov, V. G. Talalaev, P. Werner et al., "Composite system based on CdSe/ZnS quantum dots and GaAs nanowires," Semiconductors, vol. 47, no. 10, pp. 1346-1350, 2013.

[6] S. R. Cordero, P. J. Carson, R. A. Estabrook, G. F. Strouse, and S. K. Buratto, "Photo-activated luminescence of CdSe quantum dot monolayers," The Journal of Physical Chemistry B, vol. 104, no. 51, pp. 12137-12142, 2000.

[7] K. Pechstedt, T. Whittle, J. Baumberg, and T. Melvin, "Photoluminescence of colloidal CdSe/ZnS quantum dots: the critical effect of water molecules," Journal of Physical Chemistry C, vol. 114, no. 28, pp. 12069-12077, 2010.

[8] M. Oda, A. Hasegawa, N. Iwami et al., "Reversible photobluing of CdSe/ZnS/TOPO nanocrystals," Colloids and Surfaces B: Biointerfaces, vol. 56, no. 1-2, pp. 241-245, 2007.

[9] T. Uematsu, J. Kimura, and Y. Yamaguchi, "The reversible photoluminescence enhancement of a CdSe/ZnS nanocrystal thin film," Nanotechnology, vol. 15, no. 7, pp. 822-827, 2004.

[10] M. Jones, J. Nedeljkovic, R. J. Ellingson, A. J. Nozik, and G. Rumbles, "Photoenhancement of luminescence in colloidal CdSe quantum dot solutions," The Journal of Physical Chemistry B, vol. 107, no. 41, pp. 11346-11352, 2003.

[11] V. G. Dubrovskii, I. P. Soshnikov, G. E. Cirlin et al., "On the non-monotonic lateral size dependence of the height of GaAs nanowhiskers grown by molecular beam epitaxy at high temperature," Physica Status Solidi (B)_Basic Research, vol. 241, no. 7, pp. R30-R33, 2004.
[12] B. S. Neporent and S. O. Mirumyants, "Spectroscopic research of processes of the transformation of vibrational energy of the complex molecules under collisions," Optica i Spectroscopia, vol. 8, no. 5, pp. 635-642, 1960.

[13] M. Boudart and J. T. Dubois, "Stabilization of energy-rich molecules. I. Energy transfer with hydrogen," Journal of Chemical Physics, vol. 23, no. 2, pp. 223-229, 1955.

[14] B. Stevens, "The collisional stabilization of excited $\beta$-naphthylamine molecules by the paraffin hydrocarbons in the gas phase," Molecular Physics, vol. 3, no. 6, pp. 589-596, 1960.

[15] S. J. Formosinho, G. Porter, and M. A. West, "Vibrational relaxation in the triplet state," Proceedings of the Royal Society of London Series A: Mathematical and Physical Sciences, vol. 333, no. 1594, pp. 289-296, 1973. 

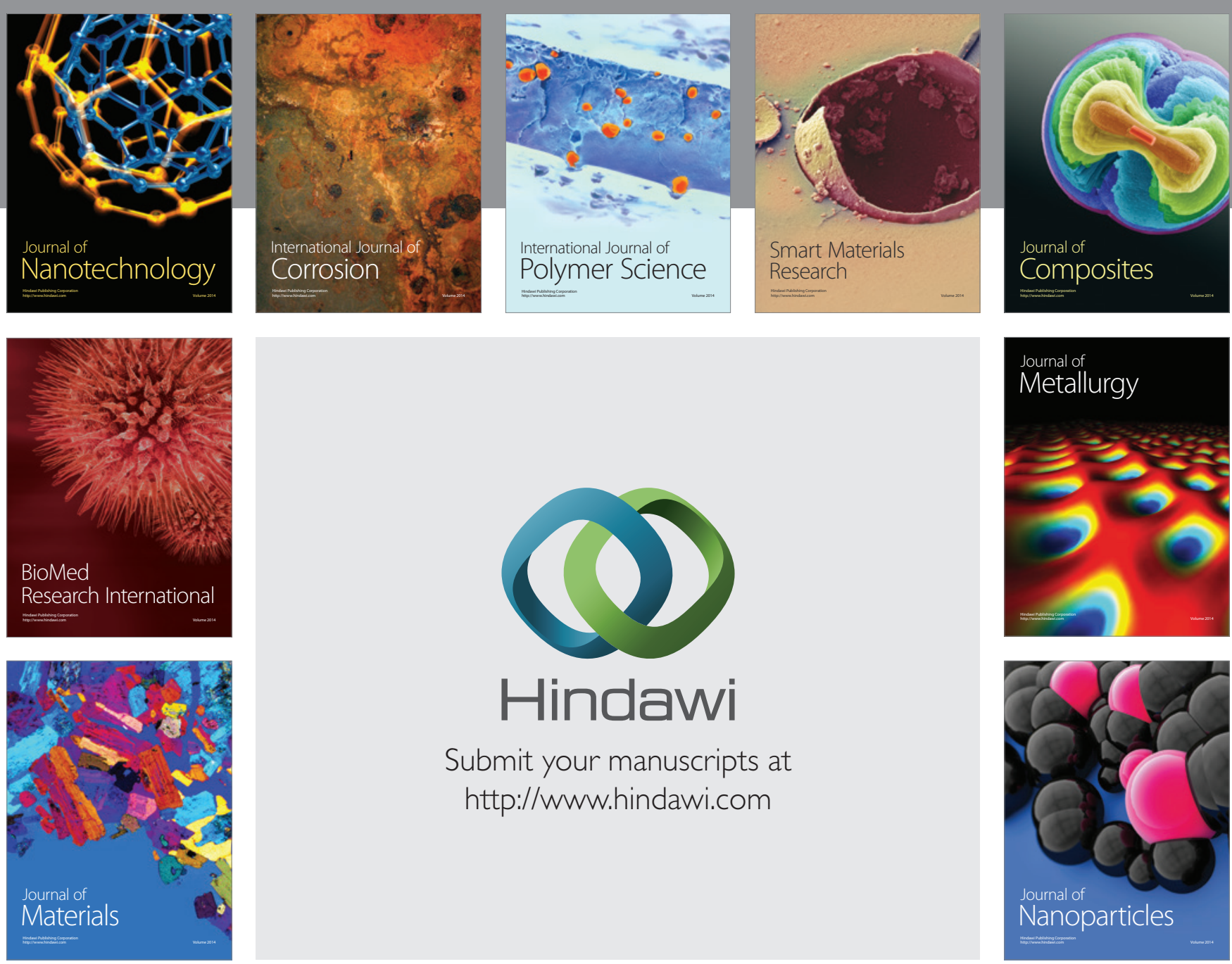

Submit your manuscripts at http://www.hindawi.com
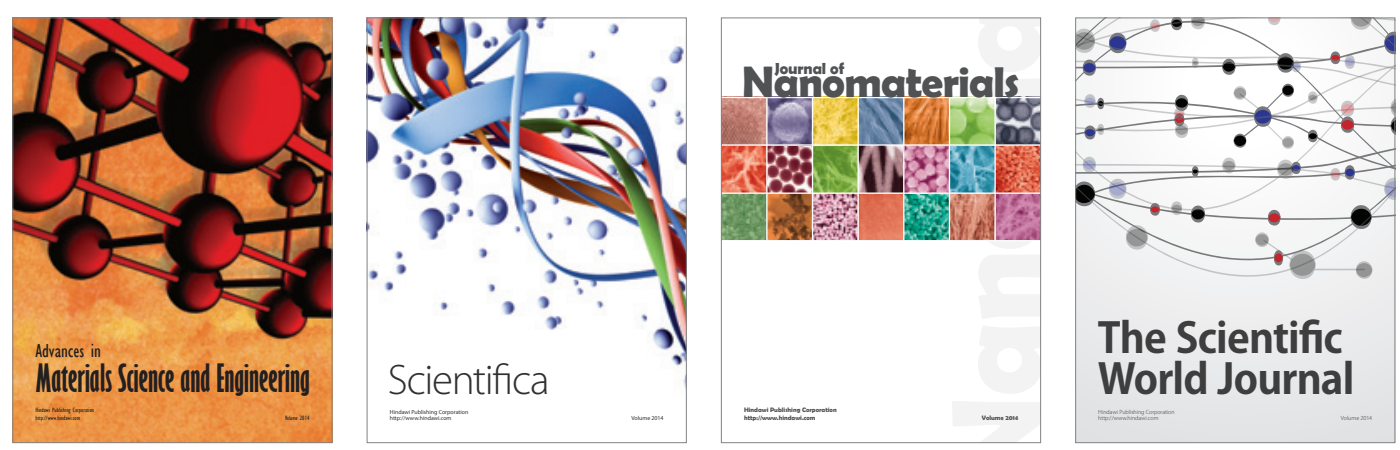

\section{The Scientific World Journal}
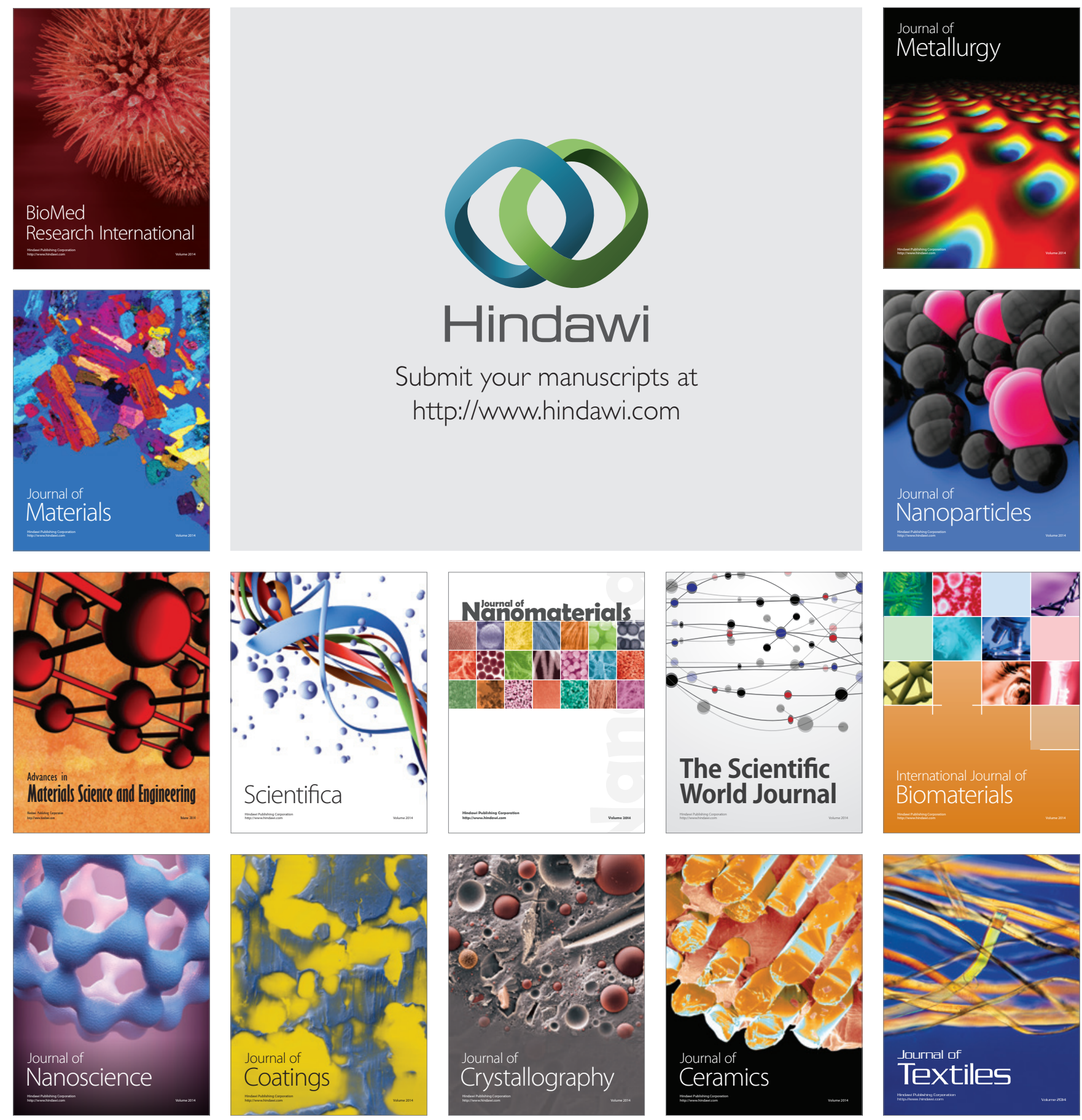